\title{
A rare cause of Emergency Department visit for melena: Gastric Mucormycoma mimicking Adenocarcinoma
}

\author{
Ivan Comelli*, Simone Bosi**, Pietro Caruana***, Giuseppe Lippi ${ }^{* * *}$, Gianfranco Cervellin* \\ * U.O. di Pronto Soccorso e Medicina d' Urgenza, Dipartimento di Emergenza-Urgenza, Azienda Ospedaliero-Universitaria \\ di Parma, Italy \\ ** U.O. di Gastroenterologia ed Endoscopia Digestiva, Dipartimento Medico Polispecialistico, Azienda Ospedaliero-Univer- \\ sitaria di Parma, Italy \\ *** U.O. Anatomia e Istologia Patologica, Dipartimento Onco-Ematologico, Azienda Ospedaliero-Universitaria di Parma, Italy \\ **** U.O. di Diagnostica Ematochimica, Dipartimento di Patologia e Medicina di Laboratorio, Azienda Ospedaliero-Uni- \\ versitaria di Parma, Italy
}

Key words: gastric mucormycosis, gastric mucormycoma, melena, gastric adenocarcinoma.

\begin{abstract}
"Mucormycosis" is an extremely rare infection typically occuring in individuals with impaired immunity. Gastrointestinal Mucormycosis is the rarest form, and shows the highest mortality rate (50-85\%). The diagnosis mostly depends on histological examination. Although culture can identify the fungal species, it is positive in only $52 \%$ of autopsy cases and $30 \%$ of surgical specimens. No reliable serologic or skin tests are available as yet. We describe a rare case of gastric mucormycoma, presenting to the Emergency Department (ED) for melena, and the potential pitfalls encountered during its evaluation and treatment. In our patient gastroscopy showed a large infiltrative and ulcerated mass located in gastric fundus and extended up to the gastro-esophageal junction. Hystology of bioptic specimens showed findings suggestive of gastric adenocarcinoma, leading the patient to surgery, consisting of total gastrectomy with esophagodigiunal and enteroenteric anastomosis. Our patient had no typical risk factor for Mucormycosis, and we suppose that the feeding by nasogastric tube, possibly through contamination by paranasal sinuses, could have caused the disseminated fungal strains, supported by a transient immunity derangement occurred during the ICU staying.
\end{abstract}

\section{Introduction}

Acute gastrointestinal (GI) bleeding is a potentially life-threatening abdominal emergency that remains a common cause of Emergency Department (ED) presentation and hospitalization. The incidence of upper gastrointestinal bleeding (UGIB) is approximately 40-150 cases per 100,000 population per year, with a 20-30 fold increase from 3rd to 9th decade of life (1). Bleeding from the upper GI tract is approximately 4 times as common as bleeding from the lower GI tract and is a major cause of morbidity and mortality. UGIB, that represents about 80-85\% of all GI bleedings, is defined as bleeding derived from a source proximal to the ligament of Treitz. Hematemesis and melena are the most common presentations of acute UGIB, and patients may present with both symptoms. Occasionally, a brisk UGIB manifests as hematochezia. Mortality rates from UGIB are 6-10\% overall (1). The increasing aging of the population and the high prevalence of associated medical comorbidities have kept the mortality rates largely unchanged for the past 30 years, despite technologic advances in endoscopy and other minimally invasive procedures (2). Hematemesis is the presentation symptom in $40-55 \%$ of patients, including patients with coffee-ground emesis. Melena is documented in approximately $70-80 \%$ of patients, and hematochezia is documented in approximately 15-20\%. The diagnosis and therapy for nonvariceal upper gastrointestinal bleeding (UGIB) has evolved over the past 3 decades from passive diagnostic esophagogastroduodenoscopy with medical therapy to active intervention with endoscopic techniques followed by angiographic and surgical approaches if endoscopic therapy failed $(3,4)$. The underlying mechanisms of nonvariceal bleeding involve either arterial hemorrhage, such as in ulcer disease and mucosal deep tears, or low-pressure venous hemorrhage, as in telangiectasias and angiodysplasias. Approximately $80 \%$ of ulcers stop bleeding, but rebleeding or continued bleeding is associated with increased mortality; therefore, differentiating the patient with a low probability of rebleeding and little comorbidity from the patient at high risk for rebleeding with serious comorbidities is imperative. The use of various endoscopic techniques, medical therapies, and visceral angiography has progressively diminished the role of surgery in the emergent management of UGIB. Nevertheless, operative intervention still represents the most definitive intervention and remains the final therapeutic option for many bleeding lesions of the upper GI tract. Of patients who develop UGIB, 3-15\% require a surgical procedure $(2,4)$. The history and physical examination provide crucial information for the initial evaluation of a patient presenting with a GI tract hemorrhage. Leading differential diagnoses for UGIB are: gastric ulcer, duodenal ulcer, esophageal varices, gastric varices, Mallory-Weiss tear, esophagitis, neoplasm, hemorrhagic gastritis, angiodysplasia, hemobilia, pancreatic pseudocyst, pancreatic pseudoaneurysm, aortoenteric fistula $(3,4)$. Infections are not traditionally considered in the differential diagnosis of UGIB, but are still a remote possibility. In this work we describe a rare case of gastric mucormycoma causing melena as a presenting symptom to our ED. 


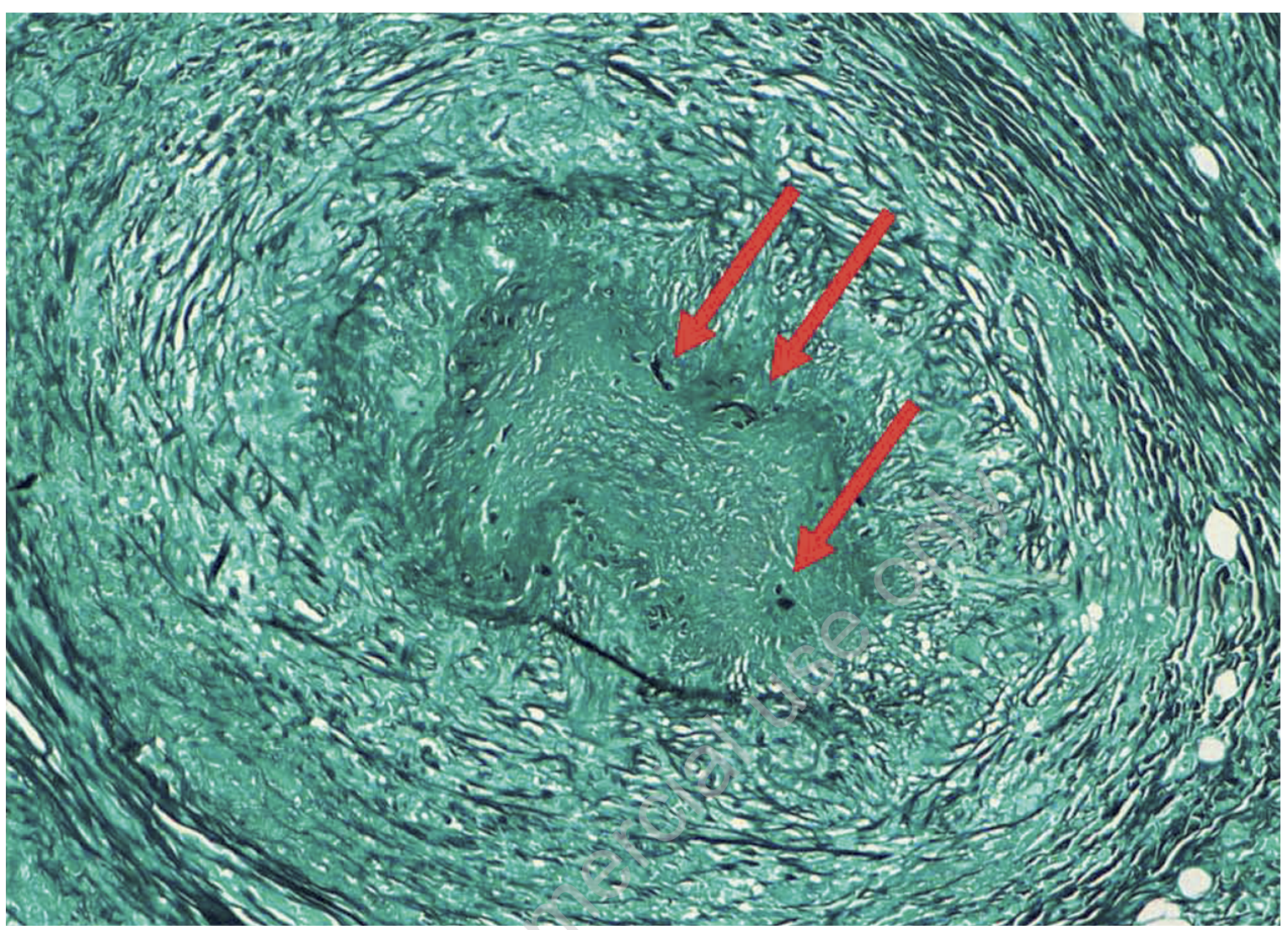

Fig. 1. Gömöri methenamine-silver staining showing a hyphae-containing-thrombus in a stomach vessel of the patient. Red arrows indicate more evident hyphae.

\section{Case Report}

A 67-year-old man with a history of arterial hypertension, monoclonal gammopathy of uncertain origin (MGUS), and recent (two months) surgery for A-type aortic dissection, was admitted to our Emergency Department (ED) for melena on March 3rd, 2010. The surgery was complicated with severe sepsis from P. Aeruginosa, which required ICU admission, mechanical ventilation, and feeding by nasogastric tube. The vital parameters were normal (blood pressure 120/80 $\mathrm{mmHg}$; heart rate 88 bpm; respiratory rate $16 / \mathrm{min}$; temperature $36.7^{\circ} \mathrm{C}$; oxygen peripheral saturation $98 \%$ ). The laboratory tests showed anemia (hemoglobin $7.6 \mathrm{~g} / \mathrm{dl}$; mean corpuscular volume $83 \mathrm{fL}$; white blood cell count 6.4x109/1; platelet count 214x109/L). A Computed Tomography (CT) scan that excluded an aorto-enteric fistula was followed by gastroscopy that showed a large infiltrative and ulcerated mass $(40 \times 35 \mathrm{~mm})$ located in gastric fundus and extended up to the gastro-esophageal junction. Hystology of bioptic specimens showed findings suggestive of gastric adenocarcinoma. This, along with the endoscopic appearance of the lesion, led the patient to surgery, consisting of total gastrectomy with esophagodigiunal and enteroenteric anastomosis. The subsequent hystological analysis showed a gastric mucormycoma with the typical angioinvasion by the fungi (Fig. 1). Cultures and identification of the fungal species were unavailable. The patient was discharged on the 7th day. According to the Infectious Diseases consultant, no antifungal therapy was administered. At present, one year after presentation at the ED, the patient is in good health, without symptoms that might suggest relapses of disease and refuses to undergo follow-up gastroscopy.

\section{Discussion}

The term "Mucormycosis" (sometimes reported as "Zygomycosis") refers to different diseases caused by Mucorales, such as Rhizopus, Mucor, Absidia, and Cunninghamellaceae. The most common agents of Mucormycosis are the Rhizopus species, 
which are more virulent than others (4). These extremely rare infections typically occur in individuals with impaired immunity, mostly with derangement of leukocyte function, due to the low virulence of the microorganisms (5). In a review of 929 patients with Mucormycosis, diabetes was the most frequent underlying risk factor (36\%), followed by malignancy (17\%), solid organ transplantation (7\%), desferroxamine therapy (6\%), and bone marrow transplantation (5\%)(6). It also occurs in patients with severe malnutrition, amoebic colitis, typhoid fever, pellagra and kwashiorkor. The recent introduction of immunosuppressive drugs and the use of voriconazole (not active against Mucorales) for the prophylaxis of opportunistic fungal infections have been suggested as risk factors for developing Mucormycosis (7).

Mucormycosis manifests in different clinical forms, namely rhinocerebral, pulmonary, cutaneous, gastrointestinal, and disseminated/miscellaneous. Gastrointestinal Mucormycosis is the rarest form ( $7 \%$ of all cases), and involves most commonly the stomach (57.5\%), followed by colon (32.3\%) and ileum (6.9\%) (6,7). The infection may arise from ingestion of fungal spores on food or contaminated sputum. The mortality rate is high (50-85\%), being the highest in patients with gastrointestinal localization (7). A high level of clinical suspicion is needed to diagnose this rare condition, so that persistent severe abdominal pain in a patient with neutropenia should alert the clinicians on the possibility of this invasive fungal infection. The diagnosis mostly depends on histological examination for the presence of hyphae. More than 94\% of sampled tissues also show infarction and angioinvasion (8). Although culture can identify the fungal species, it is positive in only $52 \%$ of autopsy cases and $30 \%$ of surgical specimens (8). This is because the infection may be localized and cannot be detected in all portions of the specimen submitted for culture. No reliable serologic or skin tests for Mucormycosis are available as yet. Recently, some have attempted to improve the diagnosis by detecting fungal nucleic acid in the serum using polymerase chain reaction (PCR) or in situ hybridization techniques.

Maravi-Poma et al. described a nosocomial outbreak of gastric Mucormycosis caused by Rhizopus microsporus in five adult patients admitted to an Intensive Care Unit (ICU). They were diagnosed with gastric Mucormycosis based on microbiological or histopathological characteristics. Upper gastrointestinal bleeding was the presenting symptom in $80 \%$ of patients. Rhizopus microsporus growth was detected in gastric aspiration samples, environmental samples, wooden tongue depressors used to prepare oral medications, and in some tongue depressors stored in unopened boxes unexposed to the ICU environment. All depressors were purchased from the same supplier. The outbreak terminated when the contaminated tongue depressors were withdrawn, and the authors conclude that wooden material should not be used in the hospital setting (9). The treatment of choice is amphotericin. The antifungal agents such as fluconazole and itraconazole, as well as newer agents such as voriconazole and capsofungin, are inactive against this fungus, whereas the new triazole antifungal agent, posaconazole, has been compared with amphotericin B, voriconazole, and itraconazole in "in vitro" studies, and was proven to be significantly more active than voriconazole and other agents. (7). Surgical resection along with amphotericin improves survival. However, the surgical option may not be feasible because of involvement at multiple sites, low platelet counts, and poor performance status. The outcome in these individuals is poor (7).

\section{Conclusions}

Our patient had no typical risk factor for Mucormycosis, and we suppose that the feeding by nasogastric tube, possibly through contamination by paranasal sinuses, could have caused the disseminated fungal strains, and the development of the disease was further supported by a transient immunity derangement occurred during the ICU staying. It is also noteworthy that this patients had a very good outcome with surgery alone and no antifungal therapy.

\section{References}

1. Fallah MA, Prakash C, Edmundowicz S. Acute gastrointestinal bleeding. Med Clin North Am 2000; 84: 1183-208.

2. Peter DJ, Dougherty JM. Evaluation of the patient with gastrointestinal bleeding: an evidence based approach. Emerg Med Clin North Am 1999; 239-61.

3. Cheung FK, Lau JY. Management of massive peptic ulcer bleeding. Gastroenterol Clin North Am 2009; 38: $231-43$.

4. Barkun A, Bardou M, Marshall JK et al. Nonvariceal Upper GI Bleeding Consensus Conference Group: Consensus recommendations for managing patients with nonvariceal upper gastrointestinal bleeding. Ann Int Med 2003;139: 84357.

5. Henderson HM, Chapman SW. Fungal infections. In: Reese RE and Betts RF Eds. A practical approach to infectious diseases. Philadelphia, Lippincot Williams \& Wilkins, 2003, pp. 720-22.

6. Roden MM, Zaoutis TE, Buchanan WL et al. Epidemiology and Outcome of Zygomycosis: a Review of 929 Reported Cases. Clin Infect Dis 2005; 41: 634-53.

7. Kontoyiannis DP, Lewis RE. Invasive zygomycosis: update on pathogenesis, clinical manifestations, and management. Infect Dis Clin North Am 2006; 20: 581-607.

8. Frater JL, Hall GS, Procop GW. Histologic features of zygomycosis: emphasis on perineural invasion and fungal morphology. Arch Pathol Lab Med 2001; 125: 375-78.

9. Maravì-Poma E, Rodiguez-Tudela JL, Garcìa de Jalòn J et al. Outbreak of gastric mucormycosis associated with the use of wooden tongue depressors in critically ill patients. Intensive Care Med 2004; 30: 724-28. 